\title{
Prevalencia de enfermedad periodontal en pacientes diabéticos tipo 2 de la Clínica de Periodoncia de la Unidad Médico Didáctica de la UAA
}

\author{
Díaz-Alfaro L*, Ortiz-Martínez CE**, González-Facio P***
}

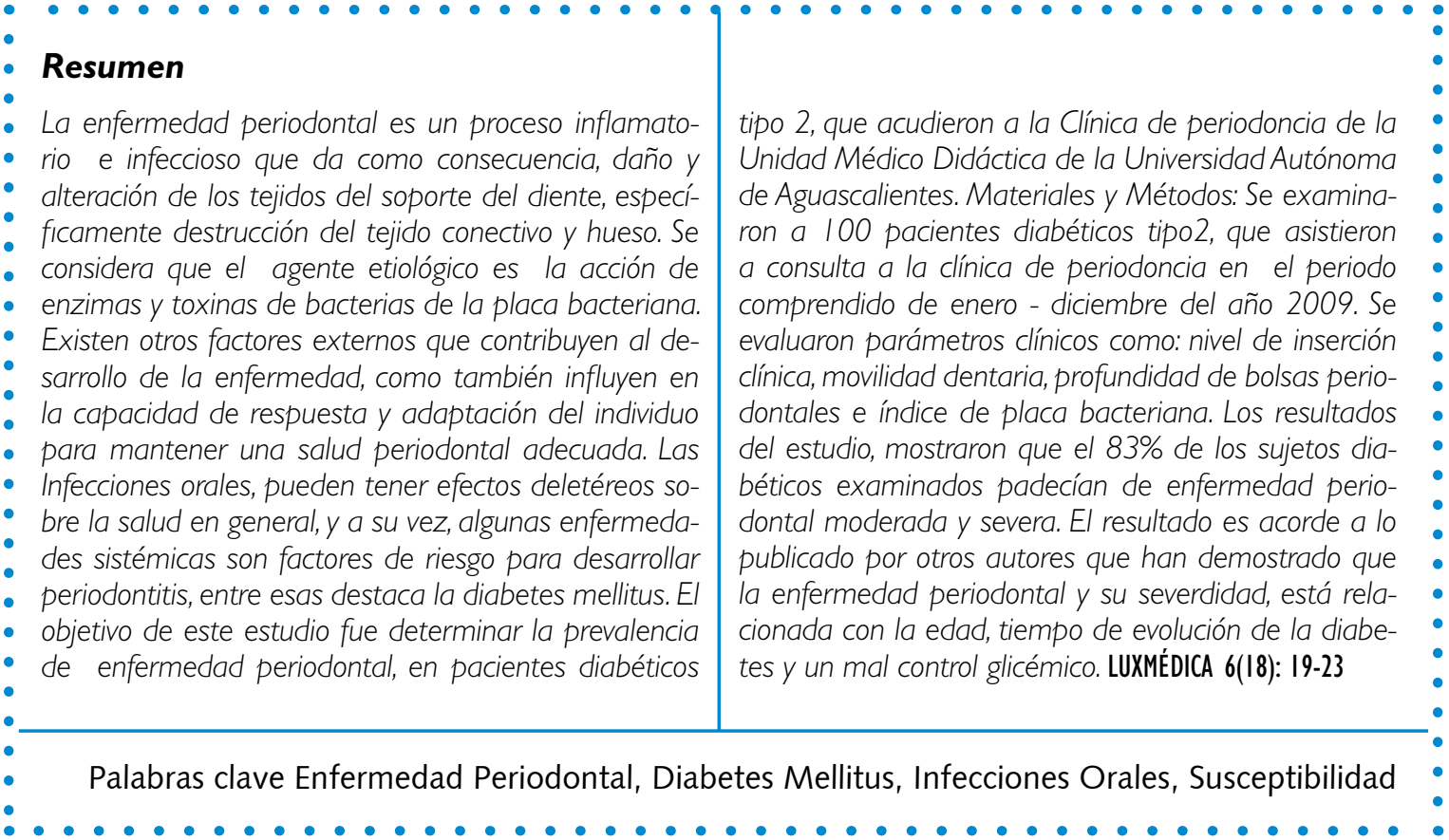

\footnotetext{
* Profesora investigadora del Departamento de Estomatología del Centro de Ciencias de la Salud de la Universidad Autónoma de Aguascalientes

** pendiente

*** pendiente

Fecha de recibido: 22 noviembre 2010

Fecha de aceptación: 30 abril 2011

Correspondencia: Lizbeth Díaz Alfaro, Centro de Ciencias de la Salud, Universidad Autónoma de Aguascalientes, Av Universidad \#940 ciudad Universitaria cp 20100 Ags., tel 9108430, correo electrónico lisaperio@hotmail.com
} 


\section{Introducción}

La enfermedad periodontal es una condición inflamatoria y de origen infeccioso de los tejidos de sostén de los órganos dentarios como lo son la encía y el hueso, que en condiciones avanzadas llegan a destruir estos tejidos, lo que conlleva a la pérdida de los órganos dentarios. ${ }^{1,2,3}$ Los agentes etiológicos primarios de la enfermedad periodontal son bacterias anaeróbicas gram negativas que ocupan el biofilm asociado a los dientes o la placa microbiana en tejidos subgingivales. ${ }^{4}$ La enfermedad periodontal causa la destrucción del tejido conectivo y hueso de soporte de los dientes, por la acción de enzimas y toxinas de bacterias de la placa bacteriana. ${ }^{4,5}$ Existe evidencia que infecciones orales, pueden tener efectos significativos sobre la salud en general, y a la vez, que algunas enfermedades sistémicas son un factor de riesgo para desarrollar periodontitis. Entre éstas destaca la diabetes mellitus. Está comprobado que la diabetes aumenta tres veces el riesgo de padecer enfermedad periodontal y que la periodontitis severa aumenta el riesgo de mal control metabólico de la diabetes mellitus. ${ }^{6,7}$. El objetivo general de este estudio fue determinar la prevalencia de la enfermedad periodontal, en pacientes diabéticos tipo 2, que acudieron a la consulta estomatológica de la Unidad Médico Didáctica.

\section{| | | | | | | | | | | | | | | | | | | | | | | | | | | | | | | | | | | | | | | | | | | | | | | | | | | | | | | | | | | | | | | | | | | | | | | | | | | | | | | | | | | | | | | | | | | | | | | | ||}

\section{Materiales y Métodos}

Se incluyeron a 100 pacientes diabéticos tipo 2, de la Clínica de Periodoncia de la Unidad Médico Didáctica de la Universidad Autónoma de Aguascalientes, en el periodo comprendido de enero a diciembre del año 2009. Todos los pacientes participaron en forma voluntaria y firmaron un formato de consentimiento informado. Los parámetros de inclusión fueron: haber sido diagnosticados como diabéticos tipo 2 y presentar un informe de laboratorio de su nivel de glucosa en ayunas, de no más de un mes de antigüedad. De los 104 pacientes iniciales, se excluyeron cuatro, por no tener la glicemia reciente.

A todos los pacientes se les realizó un examen clínico, que inició con el llenado de una ficha de evaluación periodontal utilizada en el Departamento de Periodoncia de la Universidad Autónoma de Aguascalientes.Los pacientes se examinaron, sin ha- berse cepillado, pasarse el hilo dental o hacer algún tipo de enjuagatorio.Se evaluó la profundidad de la bolsas periodontales con una sonda periodontal de $15 \mathrm{~mm}$ (N.C.), a partir del margen gingival libre y se midió en 6 superficies de cada diente (aspectos proximales y medios bucal y lingual). Una profundidad mayor de $3 \mathrm{~mm}$ se consideró patológica.

El sangrado periodontal, se evaluó 10 segundos después del sondeo periodontal y fue anotado como presente o ausente.

Las recesiones gingivales se midieron a partir de la unión cemento-esmalte hasta el margen del borde del tejido blando.

La movilidad dental evaluó como en desplazamiento en sentido buco lingual, según los criterios de Lastar. La movilidad dental se clasificó en cuatro categorías:

$$
\begin{aligned}
& 0=\text { no movilidad } \\
& 1=\text { movilidad sin desplazamiento }
\end{aligned}
$$


$2=$ movilidad con $1 \mathrm{~mm}$ de desplazamiento lateral

$3=$ movilidad con $2 \mathrm{~mm}$ de desplazamiento lateral

Las lesiones de furca fueron evaluadas con una sonda de Nabers, y se clasificaron según Rateischak (1985) midiendo su compromiso en dirección horizontal:

Clase 1: Lesión de furca con extensión de $3 \mathrm{~mm}$ al sondeo horizontal
Clase 2: El sondeo muestra una extensión de más de $3 \mathrm{~mm}$ de la lesión, pero no es completa.

Clase 3: Existe una comunicación completa.

El control de placa bacteriana se midió utilizando el índice de placa bacteriana de Löe \& Sillnes Todas las mediciones fueron realizadas por el mismo examinador. La duración de la evaluación clínica fue entre 20 y 30 minutos por paciente.

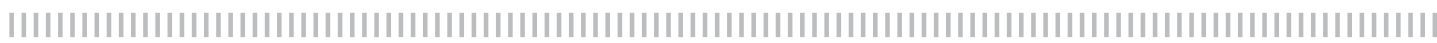

\section{Resultados}

De los 100 pacientes estudiados, la edad promedio fue de 56 años, y el rango de edad varió entre los 37 y 78 años. Entre nuestros pacientes predominó el sexo femenino en el $76 \%$ de los casos y el sexo masculino en el $24 \%$ restante.

Entre los pacientes, el tiempo promedio de la evolución de la diabetes fue de 10 años con 6 meses y la concentración de la glicemia en ayuno, en promedio, fue de $182 \mathrm{mg} / \mathrm{dl}$.

Solo en el $12 \%$ de los pacientes examinados encontramos concentraciones de glucosa en ayuno menores de 120mg/ $\mathrm{dl}$, el 31\% estaban en el rango de $121 \mathrm{a}$ $160 \mathrm{mg} / \mathrm{dl}, 37 \%$ en el rango de 161 a 200 $\mathrm{mg} / \mathrm{dl}$ y el $20 \%$ contaba con concentraciones mayores de $200 \mathrm{mg} / \mathrm{dl}$.

Se encontró también que el $96 \%$ de los sujetos examinados nunca antes habían recibido un tratamiento periodontal.

En el examen oral se encontró que el $68 \%$ de los sujetos estudiados contaban con una ausencia de 10 o más órganos dentarios y que el $98 \%$ presentaban bolsas periodontales, con mediciones que oscilaban entre 4 y $12 \mathrm{~mm}$ de profundidad.

En la evaluación de sangrado periodontal se encontró que el $91 \%$ de los sujetos presentaban hemorragia en por lo menos 6 dientes.
El $96 \%$ de los pacientes evaluados mostraron evidencia de movilidad dental, la cual oscilaba en un grado 3.

Las recesiones gingivales estuvieron presentes en $88 \%$ de los sujetos examinados, con longitudes entre 2 y $8 \mathrm{~mm}$.

La presencia de lesiones de furca estuvo presente en $71 \%$ de los casos.

Los resultados para el índice de placa bacteriana mostraron que el promedio para este índice fue de $85 \%$. Se encontró también, que el estado periodontal era más severo en las áreas de molares y de incisivos inferiores. Se encontró según las normas de la Academia Americana de Periodoncia, que el $14 \%$ de los sujetos examinados presentaban una periodontits leve, el $44 \%$ una periodontitis moderada, y el $39 \%$ una periodontits severa y solamente $3 \%$ de los sujetos examinados mostraron ausencia de enfermedad periodontal las cuales poseían los resultados más bajos de glucosa en ayunas.

Se observó también que el $47 \%$ de los pacientes que padecían de una periodontits moderada-severa, contaban con concentraciones de glucosa en ayunas mayores a $180 \mathrm{mg} / \mathrm{dl}$ y los pacientes a los que se les había diagnosticado la diabetes hacía más 10 años (42\%), todos contaban con una periodontits moderada ó severa. 


\section{Discusión}

Los datos obtenidos en nuestra investigación muestran una alta prevalencia de enfermedad periodontal en los pacientes diabéticos, resultados que son compatibles a lo reportado por otros autores, donde han encontrado que existen una relación estrecha entre la diabetes mellitus y la enfermedad periondotal. Investigaciones realizadas en los indios Pima han demostrado que los diabéticos tiene tres veces más riesgo de padecer enfermedad periodontal severa que los no diabéticos, independientemente de la edad, sexo o higiene dental de los pacientes.

Los resultados generales de este grupo, muestra sustancialmente una mayor prevalencia de enfermedad periodontal severa en pacientes con diabetes mellitus tipo 2. La prevalencia de periodontitis severa fue igual para hombres y mujeres, con una mayor prevalencia en personas de mayor edad.

Este último factor también se pudo corroborar en nuestro estudio, ya que los sujetos mayores de de 55 años mostraron un mayor severidad de enfermedad periodontal. Otro aspecto importante a considerar es el tiempo de evolución de la diabetes, ya que se ha demostrado que a mayor tiempo desde el diagnóstico de la diabetes, peor será la condición periodontal del paciente.

En nuestro estudio se encontró que pacientes con mayor tiempo desde el diagnóstico (más de 10 años) de la diabetes mostraban mayor severidad de enfermedad periodontal.

Los hallazgos clínicos que soportan esta evidencia, demuestra que existe una alta prevalencia de patología periodontal, entre estos, podemos mencionar la pérdida de adherencia como resultado de bolsas periodontales mayores de $4 \mathrm{~mm}$ (alcanzando en algunos casos hasta $12 \mathrm{~mm}$ de profundidad), características que estuvie- ron presentes en altos porcentajes en los sujetos de estudios.

Ervasti $^{8}$ y colaboradores en estudios realizados en pacientes diabéticos con un control metabólico pobre, encontraron un aumento en el sangrado periodontal en comparación con sujetos no diabéticos. Sastrowijo 9 reportó que el sangrado periodontal en diabéticos es similar al de las personas no diabéticas, si existe un control glicémico adecuado. En nuestra investigación se demostró que el $91 \%$ de los sujetos examinados mostraban sangrado periodontal, los cuales el $88 \%$ poseían un control glicémico pobre o por arriba de los parámetros normales $(120 \mathrm{mg} / \mathrm{dl})$.

Es reconocido que los pacientes diabéticos sufren de alteraciones en los tejidos y en sus defensas, como lo es el impedimento en la función de los leucocitos polimorfonucleares y el metabolismo del colágeno, lo cual los hace más susceptibles a infecciones y por lo tanto tener un mayor impacto en la severidad de la periodontitis. Nishimura ${ }^{10}$ ha reportado que los niveles altos de glucosa disminuyen la migración de células del ligamento periodontal, lo que compromete la reparación y regeneración en la enfermedad periodontal.

Según Grossi ${ }^{11}$ en estudios realizados en pacientes diabéticos, la salud periodontal ayuda en el control glicémico. Es por eso que el control glicémico riguroso es necesario para evitar complicaciones periodontales, como también es de suma importancia, un adecuado cuidado periodontal para ayudar en el control de la glicemia. 


\section{Conclusiones}

La prevalencia de la enfermedad periodontal en los pacientes diabéticos tipo 2, que fueron tratados en la clínica de Periodoncia de la Unidad médico didáctica de la UAA, concuerda con otros estudios.

La severidad de la enfermedad periodontal en los pacientes con diabetes, está relacionada con la edad y con el tiempo desde el diagnóstico de la misma. Los niveles de glucosa por arriba de lo normal (presentes en $88 \%$ de los sujetos estudiados), demuestra que el estado periodontal empeora a mayores niveles de glucosa en sangre, y también disminuyen la capacidad del organismo de reparar y regenerar las lesiones periodontales debido a su condición sistémica. Por otra parte, la infección periodontal activa presente en estos individuos dificulta el control glicémico.

Se concluye que la mayoría de los sujetos examinados (83\%) en nuestro estudio padecían de una enfermedad periodontal moderada o severa y que la mayoría de estos sujetos desconocen la importancia de la prevención y el cuidado periodontal que ellos necesitan debido a su condición sistémica. Esto se refleja, en que la mayoría, no busca ayuda profesional para prevenir las serias complicaciones en su salud oral.

\section{Bibliografía}

1 Brian L, Mealey J Moritz. Hormonal influences, effects of diabetes mellitus and endogenous female sex steroid hormones on the periodontium, Periodontology, 2000,9(32): 59-68.

2 Heitz Mayfield LJA. Disease progression: identification of high-risk groups and individuals for periodontitis. J. CLIN PERIODONTOL,2005, 32(6):196-209.

3 Zermeño Ibarra J. Fundamentos de la cirugía periodontal. $1^{a}$ Edición: México, Editorial Universitaria Potosina. 2003.

4 Newman T. Periodontología Clínica, 10a. edición: 2009. México: McGraw Hill.

$5 \mathrm{Ku} \mathrm{Lu}$, Pau Chyu Yang. Cross-Sectional Analysis of Different Variables of Patients with Non-Insulin Dependent Diabetes and Their Periodontal Status; The International Journal of Periodontics and Restorative
Dentistry. 2004;10(24):70-79.

6 GOLDMAN \& COHEN; (1993) Periodoncia $3^{a}$. Edición, México: Interamericana;

7 Per Axelsson. Diagnosis and Risk Prediction of Periodontal Diseases; Quintessence Publishing CO. 2002;13(16):302-312.

8 Ervasti et al. Diabetes A Risk Factor for periodontitis in Adults . J Periodontol 2007;530-538.

11 Grossi S. Treatment of periodontal disase in Diabetes Reduces glycated hemoglobin. J.Periodontol ; 2007: 713-719.

9 Sastrowijo Type 2 diabtes mellitus and periodontal disease J.Am Dent Assoc.;2008.121: 532-536.

10 Nishimura Long Term control of diabetes mellitus and periodontitis.JClin.Periodontol;2006:431-435 